\title{
Palatin (Rome)
}

Fouilles de la Vigna Barberini

\section{Françoise Villedieu}

Surintendance archéologique de Rome et en collaboration avec le Centre Camille Jullian et l'École française de Rome (éd.)

\section{(2) OpenEdition}

Journals

Édition électronique

URL : http://journals.openedition.org/cefr/318

DOI : $10.4000 /$ cefr.318

ISSN : 2282-5703

Éditeur

École française de Rome

Référence électronique

Françoise Villedieu, «Palatin (Rome) », Chronique des activités archéologiques de l'École française de Rome [En ligne], Italie centrale, mis en ligne le 19 décembre 2012, consulté le 02 mai 2019. URL :

http://journals.openedition.org/cefr/318; DOI : 10.4000/cefr.318

Ce document a été généré automatiquement le 2 mai 2019.

(c) École française de Rome 


\title{
Palatin (Rome)
}

\author{
Fouilles de la Vigna Barberini
}

\section{Françoise Villedieu}

Surintendance archéologique de Rome et en collaboration avec le Centre Camille Jullian et l'École française de Rome (éd.)

1 En 2011, les activités de l'équipe qui intervient sur le site de la Vigna Barberini se sont développées dans deux directions, l'une orientée vers la publication des recherches menées avant 1999, l'autre vers l'exploitation des données recueillies depuis 2009.

\section{Les fouilles avant 1999}

\section{- Présentation des travaux réalisés de 1985 à 1998}

Le texte d'une communication consacrée aux fouilles réalisées de 1985 à 1998 sur le Palatin, par l'École française de Rome en collaboration avec la Soprintendenza archeologica, devrait paraître dans les actes de la rencontre organisée en 2010 à Rabat en Hommage à la mémoire de Maurice Lenoir ${ }^{1}$.

- Préparation d'un volume sur les soutènements de la terrasse

3 Par ailleurs, l'exploitation des données de ces fouilles anciennes s'est poursuivie à travers la préparation du volume portant sur les soutènements antiques. Au cours des trois dernières années, cette tâche a souffert de la concurrence exercée par les découvertes récentes, sur lesquelles se sont concentrés nos efforts. En 2011, toutefois, les moyens accordés à l'équipe par l'École française de Rome ont été employés principalement pour financer le travail d'une dessinatrice chargée de préparer les illustrations de cette étude. Fin 2011, les plans, élévations, coupes et restitutions qui doivent illustrer l'étude des soutènements sont achevés, ce qui va permettre de reprendre les textes déjà rédigés, de les compléter en intégrant les observations que suggère la mise en forme graphique, d'ajouter les renvois. À la suite de cette confrontation entre textes et dessins, il sera certainement nécessaire d'apporter quelques modifications aux seconds, que ce soit pour corriger des interprétations ou ajouter des commentaires susceptibles de faciliter la lecture. En outre, il faut prévoir l'intervention d'une personne capable de régler les problèmes pratiques que pose le traitement des images par l'imprimeur, des problèmes 
qui dérivent principalement de la nécessité où l'on se trouve de réduire radicalement des dessins riches en détails, afin d'adapter des constructions de grandes dimensions à la taille d'un livre. Un mois de travail d'un architecte connaissant bien AutoCad s'impose pour réaliser ces deux tâches, tandis qu'il faudra encore consacrer trois mois pleins à la rédaction pour conclure l'ouvrage et être en mesure de le livrer à l'éditeur.

\section{Fouilles de la cenatio rotunda (2009-2010) ${ }^{2}$}

Durant cette année, les recherches sur le terrain ont été suspendues provisoirement par la Soprintendenza speciale per $i$ beni archeologici di Roma, afin d'inclure la fouille dans le parcours offert aux visiteurs de l'exposition Nerone $e^{3}$. Dans ce but, l'espace qui avait été ouvert en 2010, à l'est de l'aire de fouille antérieure et où les niveaux néroniens n'avaient pas encore été atteints, a été rebouché et divers aménagements ont été réalisés. Il a fallu en particulier porter remède aux dégâts provoqués par les intempéries, la couverture réalisée en 2010 n'ayant pas été dotée de gouttières. Des étaiements ont été refaits à l'intérieur du secteur de la construction néronienne actuellement mis au jour et un mur fait de parpaings de tuf a été dressé sur la limite orientale de l'espace présenté au public.

Les activités de l'équipe scientifique ont donc été dirigées vers des tâches visant à exploiter les données recueillies durant les campagnes de 2009 et 2010, et à valoriser les premiers résultats de la fouille dans le cadre de communications, conférences et articles. En parallèle, les efforts ont porté également sur la recherche de financements complémentaires, utiles pour poursuivre tant l'enquête sur le terrain, que l'exploitation des résultats.

6 En 2011, l'équipe a bénéficié du support du Centre Camille Jullian, de celui de la Soprintendenza, qui a permis de rétribuer le travail de deux des personnes qui ont commencé à dresser l'inventaire du mobilier et, enfin, de celui de l'Université de Provence, qui a accordé un financement de $10.000 €^{4}$; ces moyens se sont donc ajoutés à ceux qui nous ont été accordés par l'École française de Rome, réservés toutefois, en majorité, aux activités décrites plus haut.

\section{Exploitation des données recueillies durant les campagnes de 2009 et 2010}

7 Dans un premier temps, la responsable de la fouille a repris et analysé les données des deux campagnes, en particulier pour construire un diagramme stratigraphique, qui expose sous une forme synthétique tous les épisodes de l'histoire du site. Le diagramme a fourni des repères chronologiques utiles pour coordonner et structurer les opérations décrites ci-dessous.

Trois membres de l'équipe, Marta Fedeli, Laura David et Stéphane Abellon ont ensuite entrepris de dresser l'inventaire du mobilier archéologique issu des mêmes campagnes de fouille. L'opération, qui a duré quatre mois, a porté en priorité sur le mobilier des strates les plus significatives, à savoir celles qui correspondent au remblaiement de la construction néronienne, aux aménagements du palais impérial flavien, ainsi qu’à ses phases de transformation et de vie, puis à la réalisation du grand sanctuaire sévérien (soit, au total, 93 strates). Ont été laissés en attente, pour l'heure, les mobiliers recueillis dans les couches archéologiques qui se sont formées à compter de la fin de l'Antiquité. Il 
convient de souligner que la moitié du temps indiqué, environ, a été employée pour traiter les milliers de tessons d'objets en céramique trouvés dans un réseau d'égouts, ces vestiges étant constitués de fragments qui souvent pouvaient être regroupés et recollés sur la base de leur appartenance à un même vase. Le mobilier inventorié a été rangé en fonction de la datation des strates et de la nature des objets concernés, de manière à faciliter le travail des spécialistes qui devront intervenir pour l'étudier.

Cette étude, qui représente la seconde phase du traitement, a commencé cette année, mais elle n'a porté que sur les objets en verre, les vases à parois fines et les restes de faune particulièrement abondants au sein du mobilier mis au jour dans les égouts antiques. Les missions des experts auxquels ces objets ont été confiés ont été prises en charge par le Centre Camille Jullian dans un cas, par l'Université de Provence pour les trois autres.

L'examen des 1.165 fragments de verre recueillis dans un court tronçon des canalisations du palais impérial a permis à Danièle Foy d'individualiser 170 pièces, correspondant vraisemblablement, au maximum, à une centaine d'individus. L'ensemble est très homogène, plus de $90 \%$ des verres étant réalisés dans une même matière incolore et plus de 50 \% relevant de la même forme (gobelets cylindriques ou ovoïdes à pied annulaire). La plupart des vases appartiennent à des types ordinaires, très répandus à la fin du $\mathrm{II}^{\mathrm{e}}$ siècle et au début du III siècle dans les provinces occidentales. Seules quatre ou cinq pièces peuvent être classées dans la catégorie de la vaisselle de luxe.

11 Le bilan du traitement des restes de vases à paroi fine, dont s'est chargée Julie Leone (doctorante AMU), est similaire. Le total de 369 fragments correspondant à, au minimum, 57 individus se répartit entre des formes très largement diffusées entre la fin du $\mathrm{II}^{e}$ et le début du III siècle. Cinq pièces ornées de perles de pâte de verre et d'un fin décor à la barbotine, dont un petit bol intact, se distinguent dans ce cadre par leur aspect plus précieux et leur appartenance à une production dont la diffusion est plus restreinte.

Parmi les informations que livre l'examen de la faune, certaines contribuent à préciser l'interprétation que l'on peut avancer à propos du contexte dans lequel se conservaient les restes. Ainsi, le caractère extrêmement homogène du mobilier a permis d'établir que les dépôts retrouvés dans les égouts se sont formés en un temps très court. Ce mobilier semble correspondre à un unique épisode de vie du palais, ce qui confirme le sentiment que suggérait la fouille, à savoir que d'une part les égouts servaient à éliminer une bonne quantité des déchets produits par les habitants du palais et, d'autre part, que cet usage des égouts était rendu possible par le fort débit des eaux qui y circulaient, ainsi que par un entretien constant des conduits que l'on devait prendre soin de curer régulièrement. Par ailleurs, Sébastien Lepetz observe qu'une proportion écrasante des os recueillis provient de têtes de porcs qui portent des traces évidentes du travail réalisé par un charcutier. Il s'agirait donc de restes de préparation culinaires. Cette interprétation est confirmée par les traces d'ouverture à cru dont Myriam Sternberg a relevé la présence sur les très nombreuses coques de tellines retrouvées. En revanche, rares sont les restes témoignant de la consommation de mets cuisinés, des restes qui ont donc été rejetés ailleurs.

13 Parmi les espèces identifiées au sein des 5.628 fragments de faune terrestre examinés par le premier chercheur, le porc est donc largement prédominant, à côté d'espèces relativement communes (mouton, chèvre, coq, bœuf, oie) et d'autres plus rares (belette ou hermine et lion, ce dernier étant représenté par un seul os, isolé dans un contexte du II 
e siècle). Au sein des 2.344 fragments de faune aquatique, examinés par la seconde spécialiste, ce sont les valves de tellines qui sont de loin les plus nombreuses.

En parallèle, un jeune architecte, Jérémy Schodet, a commencé à mettre au net les relevés effectués sur le terrain, en les organisant en fonction de la séquence stratigraphique. Sur ce front, le travail est bien avancé et les informations déterminantes pour la connaissance du bâtiment néronien ont été traitées. En revanche, la mise au net des plans par phase pour les épisodes succédant à l'époque néronienne n'est pas achevée. En effet, ce collaborateur a consacré une grosse part de son temps de travail, assuré par le financement de l'Université de Provence et par l'École française de Rome, à réaliser des reconstructions utiles pour présenter nos observations et nos hypothèses touchant l'interprétation de la cenatio rotunda (voir les figures 1-3).

Fig. 1 - Palatin. Restitution de l'orographie du terrain, qui est maintenant masquée sous la terrasse artificielle qui a remodelé le relief à partir des années 70 du ler siècle.

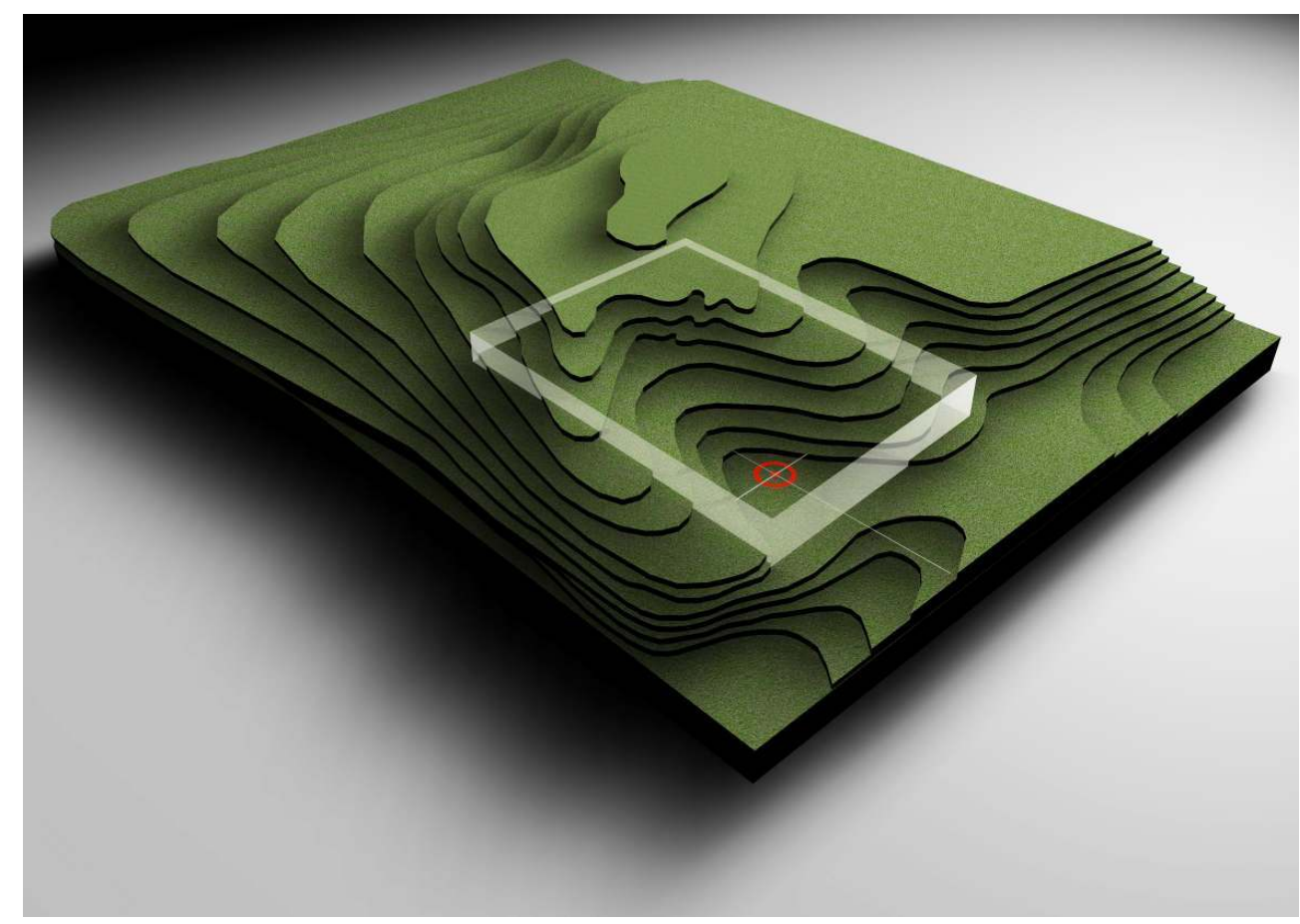

Sur la maquette, le cadre blanc délimite cette terrasse, tandis que le cercle rouge représente la construction néronienne.

Dessin J. Schodet. 
Fig. 2 - Palatin. Restitution hypothétique des planchers superposés au soubassement néronien.

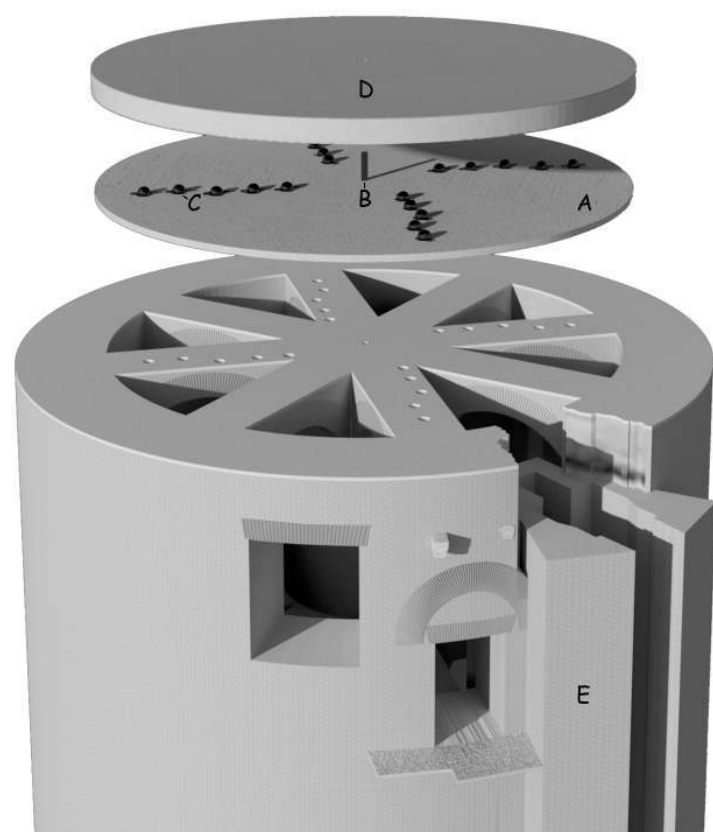

N'ayant pas retrouvé de traces de maçonneries au sommet du bâtiment, nous supposons qu'il était couronné par un plancher fixe A, percé par la cavité B, au centre, ainsi que par des ouvertures circulaires correspondant aux cavités hémisphériques $C$. Ces dernières accueillaient des sphères fixées sur le plancher A. Dans la cavité B venait se loger le pivot du plancher mobile D. La rotation de ce dernier, si elle était assistée par ces précurseurs de nos roulements à billes, était nécessairement assurée par un mécanisme, dont nous proposons de reconnaître les traces dans le secteur E qui comprend un court tronçon du mur annulaire et l'appendice méridional.

Hypothèse F. Villedieu, mise en forme graphiquement par J. Schodet, 2010. 
Fig. 3 - Palatin. Essai de restitution de la salle à manger de Néron, la cenatio rotunda placée au sommet du soubassement mis au jour par les fouilles de la Vigna Barberini.

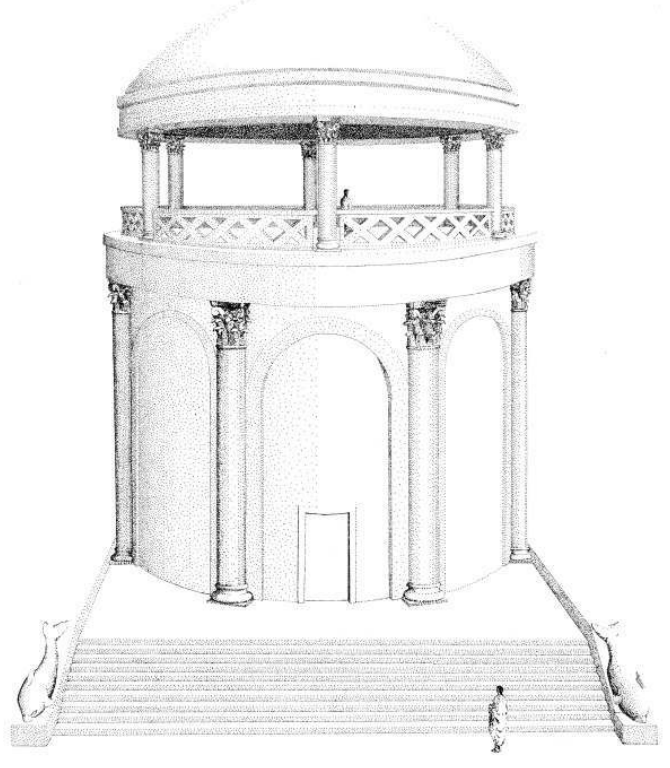

Le dessin reprend les détails que donnent les revers des dupondii portant la légende MAC AVG.

Dessin J. Schodet, 2011.

\section{Présentations : conférences, communication, articles}

La découverte du bâtiment néronien et l'interprétation que nous proposons à son sujet ont été exposées à quatre reprises durant l'année 2011, en mars à Paris, d'abord à l'INHA au cours d'une séance de la SFAC, puis à l'Auditorium du Louvre, en avril à l'Université de Fribourg et à celle de Genève. Un texte résumant la première communication est en cours de publication, de même qu'un article confié aux CRAI et un autre est paru, en ligne, dans la revue Neronia electronica ${ }^{5}$.

\section{Recherche de financements pour poursuivre l'enquête sur le terrain et pour exploiter les données recueillies}

Au stade actuel des connaissances, l'identification de la cenatio rotunda apparait comme la seule solution permettant de donner un sens aux indices réunis durant les fouilles, en particulier à tous ceux qui semblent correspondre à des pièces d'un mécanisme servant vraisemblablement à faire tourner un plancher ${ }^{6}$. Il n'en reste pas moins que l'on aimerait asseoir cette hypothèse sur une base plus solide, tandis que la curiosité que fait naître le fonctionnement du mécanisme incite lui aussi à se tourner vers le terrain pour rechercher d'autres éléments pour en restituer les détails. La poursuite de la fouille s'impose donc dans ces conditions. Les deux premières campagnes ont été financées par 
la Soprintendenza speciale per $i$ beni archeologici di Roma, qui souhaiterait être soulagée partiellement de cette charge.

Les moyens dont disposent les partenaires français associés à ce projet, l'École française de Rome et le Centre Camille Jullian, ne sont pas suffisants pour prendre en charge l'équipe scientifique qui doit suivre les travaux sur le terrain, traiter et exploiter les résultats. Nous avons donc décidé de rechercher des financements complémentaires et on peut estimer à environ cinq mois le temps que l'auteur de ces lignes a consacré cette année à cette tâche.

Les dossiers préparés étaient ou sont destinés à la Fondazione Rome, qui n'a pas retenu notre projet, à d'éventuels mécènes, qui pour l'heure ne se sont pas fait connaître, et à l'ANR, qui a écarté notre première proposition en 2011, mais nous a fortement encouragés à en faire une nouvelle, qui sera déposée en janvier $2012^{7}$.

\section{Projets pour l'année 2012}

Les fouilles devraient reprendre en 2012, après la fermeture de l'exposition " Nerone ». En conséquence, la plus grande partie, sinon la totalité, des moyens qui seront alloués à l'opération devra être réservée au suivi scientifique de l'enquête menée sur le terrain. Toutefois, nous ne suspendrons pas totalement les activités développées en 2011, nos objectifs étant de tenter de conclure la publication des soutènements et, éventuellement, de poursuivre les études portant sur le mobilier archéologique.

\section{BIBLIOGRAPHIE}

Tomei - Rea 2011 = M. A. Tomei et R. Rea (éd.), Nerone, Catalogue de l'exposition, Rome, avril-fin d'année 2011.

Villedieu à paraître = F. Villedieu, Les recherches entreprises sur la Vigna Barberini (1985-1998, Rome, Palatin), à paraître dans les actes du colloque De Rome à la Maurétanie tingitane. Hommage à la mémoire de Maurice Lenoir (Rabat, 24-26/11/2010).

Villedieu 2010 = F. Villedieu, La cenatio rotunda de la Maison Dorée de Néron, dans CRAI, 3, 2010, p. 1089-1129.

Villedieu $2011 \mathrm{a}=\mathrm{F}$. Villedieu, La fouille des vestiges de la salle à manger tournante du palais de Néron, dans Bulletin de la Société française d'archéologie classique, 42, 2011 (Séance du 5 mars 2011).

Villedieu $2011 \mathrm{~b}=\mathrm{F}$. Villedieu, Une construction néronienne mise au jour sur le site de la Vigna Barberini : la cenatio rotunda de la Domus Aurea ?, dans Neronia electronica, 1, 2011, p. 37-52 disponible en ligne http://www.sien-neron.fr/2011/11/neronia-electronica-\%E2\%80\%93fascicule-1-2011. 


\section{NOTES}

1. Villedieu à paraître.

2. Rappelons que c'est la Soprintendenza speciale per $i$ beni archeologici di Roma qui a pris la décision en 2009 de réaliser ces fouilles et qui en a assuré le financement, en collaboration avec le Commissariato. Sur cette opération, la Soprintendenza a choisi de travailler en collaboration avec l'École française de Rome et avec le Centre Camille Jullian. En 2009 et 2010, les recherches étaient placées sous la responsabilité de M. A. Tomei, à laquelle a succédé, à la direction du secteur «Foro-Palatino », son collègue R. Egidi.

3. Tomei - Rea 2011.

4. Ce financement, qui relève des FIR 2011 Actions spécifiques, a été accordé au projet «Les fouilles de la cenatio rotunda de Néron (Palatin, Rome) » présenté fin 2010.

5. Villedieu 2011a ; Villedieu 2010 ; Villedieu 2011b. Signalons, dans le même fascicule, la contribution de notre collègue de la Surintendance: M. A. Tomei, La ex Vigna Barberini e le costruzioni neroniane del Palatino, p. 25-36.

6. Ces détails ont été décrits dans la chronique de 2010 et on en trouvera une exposition plus précise dans le dernier des trois articles cités ci-dessus.

7. Le projet proposé à la Fondazione Roma s'intitulait « A pranzo dall'imperatore. Valorizzazione dei dati raccolti nel corso degli scavi svoltisi sulla Vigna Barberini (1985-1998 e 2009-2010)», la proposition présentée en réponse à l'appel de l'ANR Blanc, « Palatin. Vivre sur le Palatin et à la cour de l'empereur. Exploitation et valorisation de données archéologiques et littéraires ».

\section{INDEX}

Mots-clés : cenatio rotunda, Domus Aurea, palais, Palatin, Néron

institutions Centre Camille Jullian (Université Aix-Marseille / CNRS), École française de Rome, Soprintendenza speciale per i Beni Archeologici di Roma

\section{AUTEURS}

\section{FRANÇOISE VILLEDIEU}

Centre Camille Jullian (Université Aix-Marseille / CNRS) - francoise.villedieu[at]gmail.com 